\title{
Legal protection of the night sky in Andalusia (Western Europe)
}

\author{
David Galadí Enríquez ${ }^{1}$ and Ángela Ranea-Palma ${ }^{2}$ \\ ${ }^{1}$ Centro Astronómico Hispano-Alemán (CAHA) \\ Apartado 2010, ES-04080-Almería, Spain \\ email: dgaladi@caha.es \\ ${ }^{2}$ Consejería de Agricultura, Pesca y Medio Ambiente, Junta de Andalucía \\ Avenida Eritaña 1, ES-41071-Sevilla, Spain \\ email: angela.ranea@juntadeandalucia.es
}

\begin{abstract}
Andalusia (Spain) houses several astronomical observatories, among them the main observational facility in continental Europe: Calar Alto Observatory. In recent years, the regional government of Andalusia has been setting up a regulation to protect the natural conditions of darkness at night all over the region. This regulation includes several outstanding features and poses specific rules to protect the influence area of Calar Alto Observatory.
\end{abstract}

Spain is a federal state composed of 19 entitites called communities, three in Africa and 16 in Europe. Andalusia $\left(87268 \mathrm{~km}^{2}\right)$ marks Europe's southernmost extreme and Spain's most populous community (8.2 million people). Its official language is Spanish.

Andalusian legislation on light pollution has been evolving since 2007 in several phases and is close to being fully operational. It protects all the territory of the community (eg. max. ULORinst $1 \%$ everywhere), with special attention on the huge area of environmentally protected land and with a specific focus on major astronomical sites: Sierra Nevada Observatory (OSN) and Calar Alto Observatory (CAHA). The influence areas of these observatories have been approved and their most outstanding feature is the restriction on white and blue light inside these zones $\left(3877 \mathrm{~km}^{2}\right.$ for Calar Alto Observatory).

Those in charge of the development of this law are well aware of the need to interact to enforce the regulation successfully. To this end, the release of the law is accompanied by an unprecedented effort of communication, education, public outreach and direct action:

Dissemination: leaflets, digital documents, electronic and phone hotlines, specific actions and materials on blue- and white-light restriction. Local councils: training programs for local politicians and technicians, legal and technical support and advice, help to search for funding and financing. Universities: training sessions for university students (architecture, environmental sciences, lighting engineers). Educational community: short film and art (posters, poetry, graffiti) contests, teaching unit for secondary schools (useable worldwide). General public: awareness campaigns in many different contexts. Amateur astronomers: annual course on multi-band photometry, participation in their meetings and events, support of their awareness campaigns. Events: fairs, participation in congresses on dark-sky protection, organization of the 1st International Meeting of Administrations with Legislation on Light Pollution (2011). Measurement campaigns: multi-band CCD-based and one-band SQM-based measurement campaigns to produce the Andalusian map of sky glow for diagnosis and to assess the future effects of the law.

The Andalusian law is not only one of the most advanced, but is a very outstanding example of what can be done when administrations take light pollution seriously. All this has been done by a small team of four specialists with external help from many Andalusian and non-Andalusian individuals and entities, both public and private. 